\title{
A Proactive System for Real-time Safety Management in Construction Sites
}

\author{
Alessandro Carbonari ${ }^{1}$, Berardo Naticchia ${ }^{2}$, Alberto Giretti² and Mario De Grassi² \\ ${ }^{1}$ Polytechnic University of Marche, DACS Department, Engineering Faculty, Ancona, Italy. Tel: \\ +390712204397; Fax: +39 0712204582; Mobile: +39 3402502768, alessandro.carbonari@univpm.it \\ 2Polytechnic University of Marche, Smart Space Solutions SRL, Via Brecce Bianche, 60131 Ancona, Italy
}

\begin{abstract}
The purpose of this paper is presenting a new advanced hardware/software system, boasting two main features: first it performs real time tracking of workers' routes in construction sites; then it implements an algorithm for preventing workers to be involved in hazardous situations. This research step is part of a wider ongoing research concerning the development of a new generation of advanced construction management systems, allowing for real-time monitoring and coordination of tasks, automatic health and safety management, on-site delivering of technical information, capture of as-built documentation. Exploiting the high accuracy provided by the UWB system responsible for position tracking and successfully tested in previous research, our software interface is able to graphically reproduce (and store) the travel patterns of workers. Moreover, it constantly checks if they are accessing hazardous areas, using an algorithm based on a predictive approach: it is conceived to predict in advance whether any worker is approaching a forbidden area, in fact performing virtual fencing. This approach could be easily extended to other applications, too. Some preliminary tests simulated in the DACS laboratory are described and the obtained results discussed.
\end{abstract}

Keywords: UWB tracking system; health and safety in construction; virtual fencing; automated monitoring.

\section{Introduction}

It is well accepted that construction sites' management is a very tough task, due to a number of reasons (Naticchia et al. 2005): they are custom designed, they host the realization of very complex building projects, involving thousands of parts and components, and changes of design plans at construction time are not uncommon. Hence, human resource and facility management would need a continuous monitoring, in order to be correctly performed. In addition, their organization is very strongly related with health and safety objectives. Considering that coordinators for health and safety matters at the construction level cannot always attend construction works while they are ongoing, it can be inferred that the practical application of the prescriptions required by the health and safety plan (according to European Council Directive 92/57/EEC) are left to workers. In addition, also coordinator's enduring presence on construction sites would not be adequate to prevent accidents in very large sites, as not capable of ubiquitous control. This situation often leads to scarce application of health and safety requirements, that could be the cause of dramatic accidents.

However, a set of recently marketed technologies could provide the necessary background for developing a new generation of real-time construction management systems, which can be seamlessly integrated into the actual arrangement of the construction work (Abderrahim et al. 2005). The two main kinds of technologies are: on one side, accurate tracking technologies, that could provide in real time the position of all workers present in a construction site; on the other side, tools for behaviour capture, used to model and then predict assets' behaviour, in order to infer what is going to happen.

The good aspect of tracking technologies is that some of them are very accurate, and are able to track workers by just equipping them with such small embedded tags, that they do not interfere with ongoing activities. It obviously needs a system setup to cover all the area of interest. Considering that they will give back in real time the position of every employed resource, then it could be interfaced with a system having the task of predicting what is going to happen and analyze if it could trigger some dangerous situations. It would have the advantage of improving presently adopted approaches for safety management and optimizing resource usage and working efficiency at the same time. The tools developed for behavioural model capture would have the task of discerning dangerous situations in advance.

This paper concerns a feasibility study of a pro-active system for health and safety management in construction sites, fully described in paragraph 3 , and mainly composed by two parts: the first performing real-time position tracking, while the second providing real-time prediction of risky events. The results 
obtained from preliminary experimental tests performed in our laboratories will be described and discussed to plan further steps.

\section{State of the Art}

Despite mobile computing for production management has already been developed and reliably applied to manufacturing (Fuller et al. 2002, Brewer et al. 1999), it is almost completely missing in construction sites, due to its outdoor, heterogeneous and highly evolving nature. Some preliminary results have been obtained in construction sites through the application of standard outdoor position tracking technologies. Some researchers have developed a mechatronic helmet, equipped with a GPS antenna and a bidirectional communication system for workers' safety control (Abderrahim et al. 2005). A lot of efforts have been made to explain the main advantages that would be pursued with the adoption of mobile computing (Rebolj et al. 2001). Among them, the most important ones, devoted to the field of construction management, are: supply delivery records and progress updates directly at the jobsite; rapid communication and collaboration throughout the entire project life cycle, from financing and planning through engineering and design, procurement, construction, and facility management; systems that provide construction teams with a projectspecific extranet, whereby remote team members can access up-to-date documents.

For that reasons, the main efforts have been directed towards mobile computing technologies development and resource tracking. Within the first field of research there are some very valuable works: an integrated RFID and GPS technology for the purpose of tracking highly customized prefabricated components and avoiding delays in construction (Ergen et al. 2007); embedding RFID tags in building components to store design data, which can be passed to the people in charge of maintenance during its operational phase (Cheng et al. 2007); efficiency improvement of tool tracking and availability increase by using RFID tags (Gajamani et al. 2007).

On the other side, there have been several attempts to develop systems for continuous resource monitoring, adopting a wide range of technologies, from Rfid to GPS. However, the only one that was shown to be really capable of providing good performances for very complex places, resulted to be Ultra Wide Band: fieldwork tracking in an iron made construction showed a position accuracy significantly better than $1 \mathrm{~m}$ (Teizer et al. 2007), the same happened for reinforced concrete made construction buildings (Giretti et al. 2008). It suggests that this technology would be very suitable for safety management, were accurate localization is critical to avoid workers' access into hazardous areas, to perform collision avoidance, to prevent falls from height and a number of accidents. Other research is facing the problem from a higher perspective: for example, a Project called "SightSafety" has the main aim of developing innovative and proactive systems for automated collision detection avoidance, which is part of a more ambitious project regarding health and safety management in constructions sites (Riaz et al. 2006). Very interesting is the two tier software system the authors have developed, whose business logic has been designed to recognize whether dangerous situations take place. It is also stated that the higher the position accuracy, the better the system performs its tasks. That remarks the importance of using some very accurate location systems like UWB.

Therefore, now big interest is focused on how tracking systems could be interfaced with software systems to recognize dangerous situations and which kind of algorithms should be developed to pursue this last objective. Among all the tested approaches, there is one attempt consisting in recording time series paths (derived from UWB tracking), in order to identify those ones which are safer by the frequency workers pass across them and then signal when some workers would choose a not safe path (Teizer et al. 2008). This approach is very similar to what proposed in other fields of research, where algorithms for behavioural models are developed: they were used to forecast workers' behaviour, in order to perform risk avoidance in advance with respect to the time when risky situations are experienced (Wang et al. 2004): thus, alert messages may precede dangers. Our paper rises from such considerations and tries to propose a first software application for virtual fencing of dangerous areas in construction. It laid the basis for further development about the production of the final system release, to be installed and tested in real construction sites. 


\section{The New Proactive System}

\subsection{Overall Description}

Figure 1-a provides a scheme of the system under development, that will be made up of some hardware sub-systems, interfaced with a software application for whole management. It is expected that tracking very complex and large construction sites will be performed by installing several UWB sub-networks, connected to one service application, implementing a software tool for data and logic management. In this first period of research we are focusing on health and safety, while other features will be added later (e.g. providing "ondemand" information to personnel operating on site, automatically producing "as-built" documentation, performing real-time management even through remote control), and exploiting the same UWB localization system. This application software is thought to implement a three tier software architecture for real-time construction management (Figure 1-b). The lowest level implements sensor, localization, communication, inference and data management logics. The middle level "business logics" implements high level task oriented functionalities (e.g. when and how managing virtual fencing or collision avoidance tools, included in the lowest level, transferring those data to the high level application modules, according to users' requests). The application layer (highest level) customizes general business logic functionalities to specific application domain, such as health and safety management. In this paper we will focus first on development of the inference API, and then on the relationship between it and the position tracking module, which is part of the localization API.

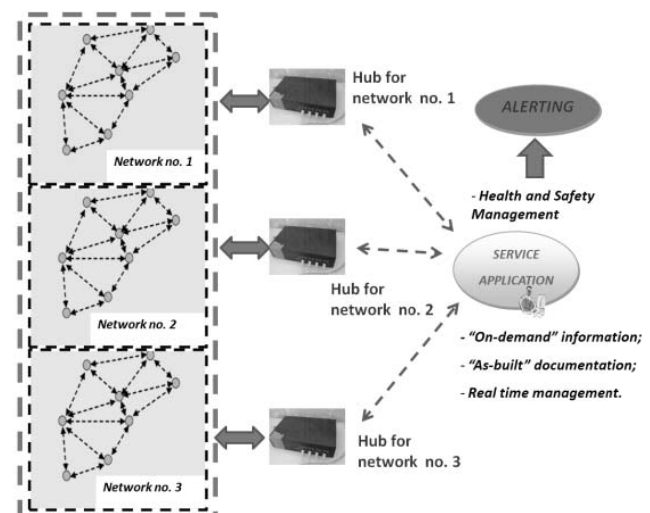

b)

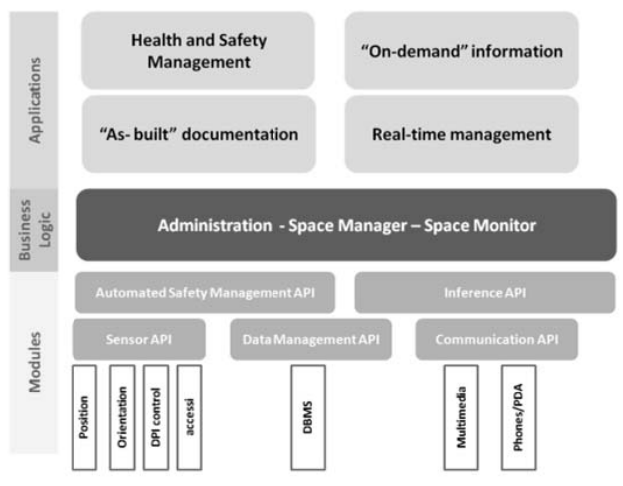

a) IL-

Figure 1. Schematization of the pro-active system logic (a) and three-tier software system implemented in the Service Application (b).

Then, a preliminary user graphic interface for the visualization of the tracked tags will be presented. For the purpose of this preliminary study, it was decided to develop a tool for the preventing workers access into previously defined dangerous areas. The algorithm is fully described in paragraph 3.3, while the graphic interface for input insertion and visualization is shown in Figure 2-a. It is subdivided into six areas: tag-user configuration, accepting user inputs and storing correspondence with their IDs; uploading map image; size and map insertion in the reference system of the monitored area; not accessible area configurations, that requires the insertion of the coordinates of the area to be not accessed; map visualization; connection to the UWB tracking system (defined in the same geographic system).

\subsection{System's Expected Performances and Previous Research}

Previous experimental tests led in the construction site shown in Figure 2-b and 2-c, let us conclude that UWB tracking systems can perform very accurate location tracking at least until reinforced concrete structure's erection (Giretti et al. 2008). Using just four receivers and equipping every resource with $1 \mathrm{~W}$ tags, it was possible to monitor a $500 \mathrm{~m} 2$ large construction area. Hence UWB overcomes the limits of GPS, which was not able to track indoor.

Instead, a different and more intense configuration for UWB receivers will be necessary to perform monitoring when masonry buildings are monitored. In any case, it came out that UWB tracking is very good for tracking from the beginning of the construction progress, until structure frame is built. Hence it would be able to support several automated features (e.g. collision avoidance, virtual fencing). 

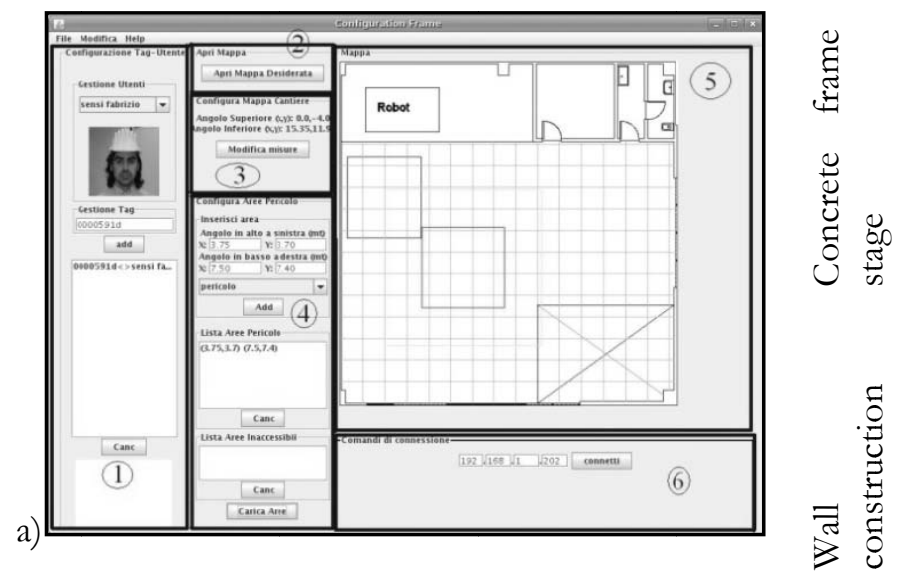

b)

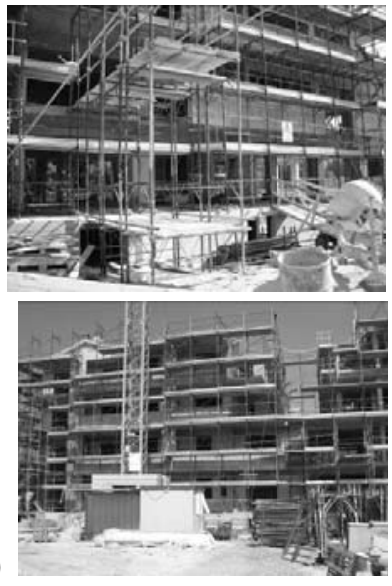

Figure 2. Graphic interface of the software system (a) and the two progress stages of the construction site used for UWB testing (b, c).

\subsection{Predictive Approach to Risk. Management}

It is generally stated that, when coping with risky situations, it is necessary to adopt a predictive approach (Wang et al. 2004). An intelligent approach is always necessary to check risky events in advance and render warning signals with suitable timing for workers, in order to prevent bad consequences. In addition, the high polling frequency of UWB technology (from 1 to $60 \mathrm{~Hz}$ ) should be exploited to process real-time data and properly manage those situations by providing alerting signals in real-time. We think that two basic intelligent approaches are available to manage those situations, whose classification is valid also in the particular case of virtual fencing of dangerous areas, considered for the purposes of this paper:

1. a deterministic approach, that predicts dangerous situations starting from the actual route stepped by workers;

2. a probabilistic approach, which could be based on probabilistic models (e.g. Bayesian Networks), to perform knowledge capture first and inference processing later, in order to predict first the route that every monitored worker is going to follow and then whether this path is expected to lead towards risky situations (Howden et al. 2003).

In this paper, we have chosen to analyze the first approach, even if the second one will be studied soon later, within the next research step. Hence, we are proposing a first predictive method to manage risks in real-time.

The software application interfaced with the UWB tracking system implements an algorithm for virtual fencing. Its main objective is checking risks in advance, in order to warning endangered operators before they could be harmed. For that purpose, the first step is discerning and localizing where dangerous areas are placed. They could be those areas were presence of workers is not allowed (e.g. close to scaffolding not equipped with protections against falling), which may be selected directly from the site layout, during the design stage. There could also be the possibility those areas are dynamically changing: in case collision avoidance between two assets is performed, one object could be processed as the area not to be entered and the other one as the operator moving throughout the construction site. Hence this first step is critical, being the basic tool for further applications. The main assumptions made for this first technologic development are:

- the dangerous area is static and its location known in advance;

- risks must be predicted before they take place.

The general approach followed in order to pursue this objective consists in surrounding every forbidden area by a "warning" strip (in our drawings, the dangerous area is usually red coloured, while the warning strip is yellow, like in the following Figure 5-a). Warning strip's thickness must be sized according to tag frequency and to average operators' speed. This second parameter was assumed to be fixed at $0.5 \mathrm{~m} / \mathrm{s}$, while the first was sized through adequate testing (paragraph 4.1).

In this way, the algorithm is able to check the behaviour of operators who have already entered the yellow area, in order to understand whether they are approaching dangers. Figure 3 better explains our algorithm's logic. It first checks whether any operator has already entered the red dangerous area: in the 
positive case a red alarm is sent, otherwise no alarms at all are sent and the algorithm checks if the operator has entered the yellow surrounding strip. If this is true, then its present position is compared with the previous one (recorded in the previous time step) and a warning alarm is sent in case he has got closer to the border line; in the opposite case the whole procedure is repeated. It is straightforward that tracking operators' movement within the yellow strip is critical for this application.

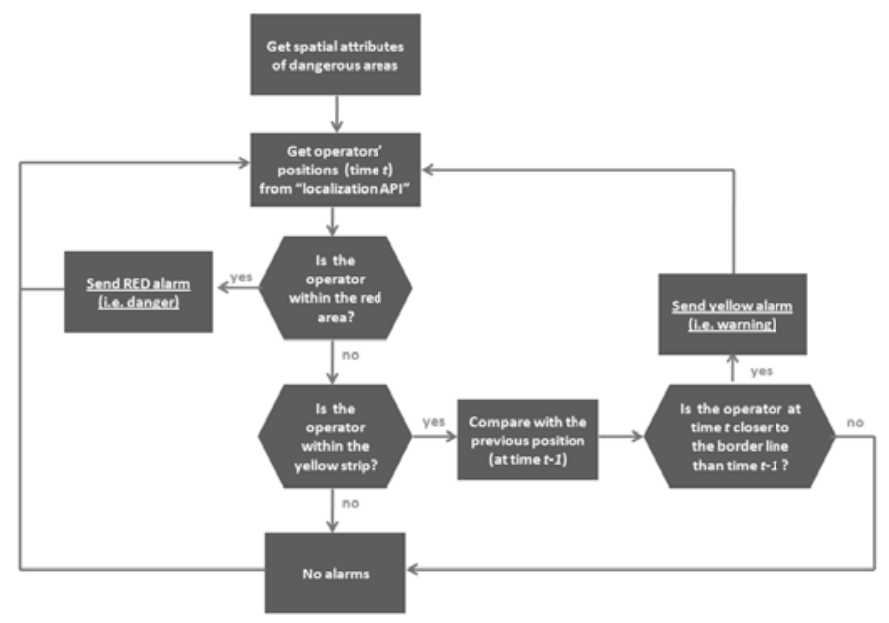

Figure 3. The algorithm's logic

\section{Experimental Campaign}

\subsection{Preliminary tests}

The experimental campaign was aimed at validating the algorithm proposed and evaluating whether the UWB system adopted is enough for the application we are suggesting in the field of health and safety management. All the tests have been performed in the laboratories of the Department of Architecture Construction and Structures (DACS) of the Polytechnic University of Marche. They have been planned in such a way to be rather representative of the actual situations expected in construction sites. Two experimental phases have been carried out:

- the first one to validate the simplified version of our algorithm, and to infer how thick should the yellow warning strip be around the red area;

- the second one to validate the final release of the proposed algorithm and conclude about its reliability.

The first set of experiments simulated the presence of a not accessible area in a construction site, and wanted to implement a reduced version of the control algorithm, made up of just the first part, signalling when a worker accessed the dangerous area. For that reasons, we measured the gap between the time when a resource crosses the border line and the time when the software alerts about that. Four UWB receivers have been placed far from the corners of one $(3.7 \times 4) \mathrm{m}$ large red area (Figure 4-b). Our hardware/software system, interfacing the UWB system's hub to our software application tool (developed in JavaTM environment) and implementing the algorithm of Figure 3, was setup. Then one of our collaborators was equipped with a $1 \mathrm{~Hz}$ tag and was asked to enter repeatedly the red area following the three paths of Figure 4-a. Measures were repeated 8 times for each path and it came out that the application tool is able to send warning signals (Figure 4-c) within a maximum distance of $1.2 \mathrm{~m}$ beyond the border line, with an average distance gap of $0.7 \mathrm{~m}$ from it. Hence a warning strip slightly wider than $1.5 \mathrm{~m}$ should be planned around the red area with $1 \mathrm{~Hz}$ tags, in order to face also rare events (e.g. some tag bursts are missing to more than one receiver and position is computed in the next iteration, in fact postponing alert signals).

Figure 4-c shows one of the red alarm signals sent by the application software after the boy simulating a worker passed beyond the border line (Figure 4-d). Our system worked very well at this stage, with no failures, being the gap determined just by the time required by the UWB system to update tags' location, which is inversely proportional to its frequency. Hence, in the following test, a $1.7 \mathrm{~m}$ thick warning strip around the red area was drawn on the laboratory's floor (Figure 5-a), simulating the strip inside which the 
algorithm checks resource's behaviour before sending a warning signal, which depends on his intention to get closer to the border line.

a

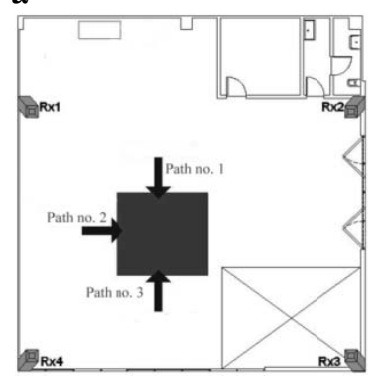

$\mathrm{b}$

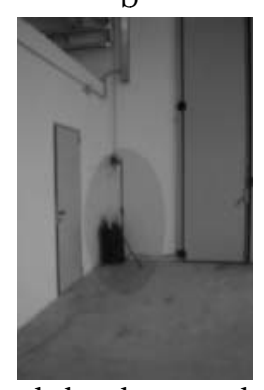

c

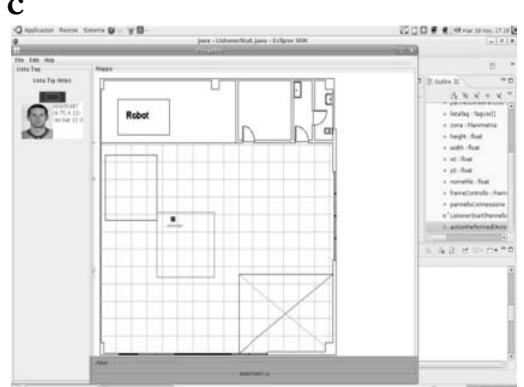

d

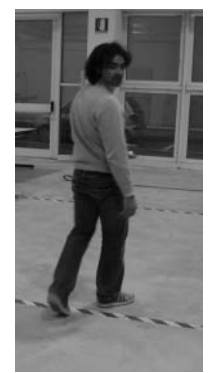

Figure 4. Red area position and the three paths used for the 1st experimental phase (a);one receiver (b); screenshot of the application sending a red alarm signal (c, d).

\subsection{Validation of the algorithm's logic}

After having arranged our laboratory as depicted in Figure 5-a, one of our colleagues was asked to step the three paths shown in figure, in order to collect 10 measures for each of them and check for errors. Three types of potential errors for the system have been preliminarily discerned:

- absence of alarms: the system does not send alarms, even if it should do.

- false red alarm: the system send red alarm even if the worker is again in the warning strip;

- false warning alarms: the system send a warning alarm even if the worker is not getting closer to the border line.

One different set of tests has been performed for each path in Figure 5-a. For path no. 1, no false alarms have been collected. For path no. 2 just one error of type 1 has been collected (due to the low tag rate of $1 \mathrm{~Hz}$, that did not allow to monitor the worker twice before accessing the red area). For path no. 3 three errors of type 1 have been collected, which were then shown to be due to interfering objects very close to receiver no. 2. Figure 5-b shows the graphic interface sending a warning "yellow" alarm, due to a worker approaching the border line.

From those results it was concluded that the algorithm's logic is correct and works properly, just in case location measures are accurate. The different performances observed among the several measures was dependent just on the random variation of location measures, proper of the UWB system. In order to better explain that concept, the worst case for the system was experimented: as in Fig 5-c, one of our collaborators was asked to walk parallel to the border line. It came out that every measure was affected by one or two errors of type 3 , due to the standard localization accuracy of UWB system, whose measures are randomly varying around the real value within a $0.3 \mathrm{~m}$ distance. Due to that, the system could send false alarms. In order to reduce that possibility, two approaches could be followed:

- increasing tags' frequency, hence reducing the yellow strip's thickness and the probability that a worker could walk parallel to the border line;

- reducing random variation around the real value, through the use of statistically filtered tracking measures.

\section{Conclusions and Future Research}

In the preliminary analyses carried out in this paper, the performances of a virtual fencing application tool have been simulated. Tests performed in our laboratories showed that our deterministic algorithm is rather reliable to perform virtual fencing of dangerous areas, using a predictive approach. Further amendments will be necessary to face also dynamic and evolving environments like construction sites, that will require to integrate our tool within a higher software level for behavioural modelling of those environments. In any case this tool is almost ready to be applied in static contexts where collision avoidance between resources must be performed. 

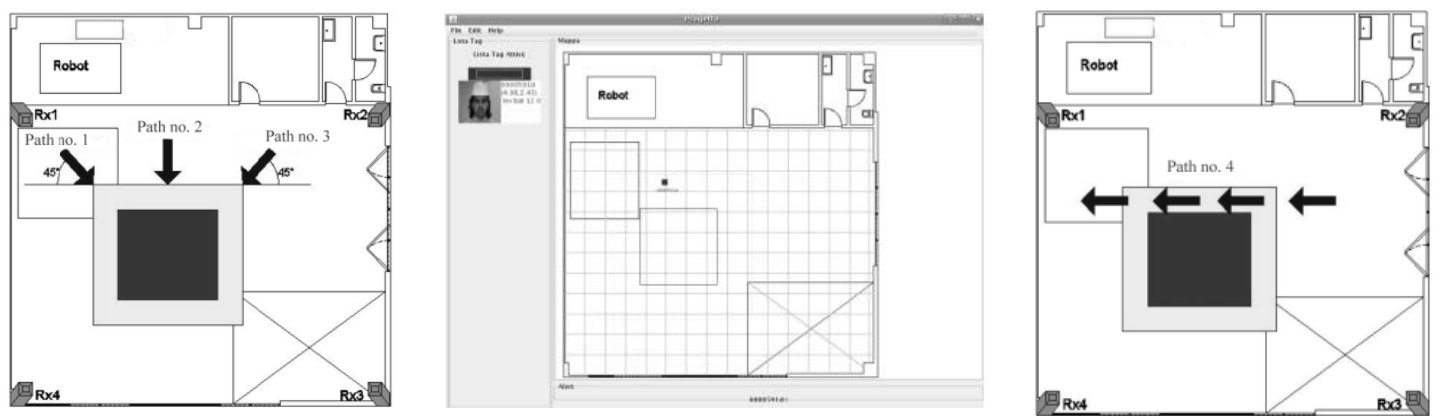

Figure 5. Laboratory setup for testing algorithm's reliability (a), screenshot of the spatial reasoning API's virtual fencing tool (b) and path no. 4 (c).

The few false alarms encountered could be solved by adopting two amendments: filtering localization data, that will minimize the random variation admitted by UWB system around the real position; using tags with a higher frequency, that would allow to decrease the warning strip's width and increase the algorithm's reliability.

Finally, other probabilistic techniques will be developed, to strengthen predictive capabilities of this application.

\section{References}

[1] Naticchia, B., Giretti, A. \& Giretti, P. (2005) Systems for Real Time Construction Site Management, Proceedings of the Joint 2006 CIB Symposium W065/W055/W086, Rome, Italy, October 2006.

[2] Abderrahim, M., Garcia, E.; Diez, R., Balaguer, C. (2005) A mechatronics security system for the construction site, Automation in Construction, Vol. 14, No. 4, 460-466.

[3] Fuller, S., Ding, Z., Sattineni, A. (2002) Case Study: Using the Wearable Computer in the Construction Industry, Proceedings of the 19th ISARC, National Institute of Standards and Technology, Gaithersburg, Maryland, September 23-25, 551-556.

[4] Brewer, A., Sloan, N., Landers, T. L. (1999) Intelligent tracking in manufacturing, Journal of Intelligent Manufacturing, Vol. 10, No. 3-4.

[5] Rebolj, D., Magdič, A., Čuš Babič, N. (2001) Mobile computing in construction. Advances in Concurrent Engineering, Proceedings of the 8th ISPE International Conference on Concurrent Engineering - Research and Applications, West Coast Anaheim Hotel, California, USA, July 28 - August 1.

[6] Ergen, E., Akinci, B., Sacks, R. (2007) Tracking and locating components in a precast storage yard utilizing radio frequency identification technology and GPS, Automation in Construction, Vol. 16, No. 3, 354-367.

[7] Cheng, M. Y.; Lien, L. C.; Tsai, M. H.; Chen, W. N. (2007) Open-building maintenance management using RFID technology, Proceedings of the 24th International Symposium on Automation and Robotics in Construction - ISARC 2007, September 19-21, Kochi, India.

[8] Gajamani, G. K., Varghese, K. (2007) Automated project schedule and inventory monitoring using RFID, Proceedings of the 24th International Symposium on Automation and Robotics in Construction - ISARC 2007, September 19-21, Kochi, India.

[9] Teizer, J.; Lao, D.; Sofer, M. (2008) Rapid automated monitoring of construction site activities using Ultra-WideBand, Proceedings of the 24th International Symposium on Automation and Robotics in Construction - ISARC 2007, September 19-21, Kochi, India.

[10] Giretti, A., Carbonari, A., Naticchia, B., De Grassi, M. (2008) Advanced real-time safety construction management system for construction sites, Proceedings of the 25th International Symposium on Automation and Robotics in construction, Vilnius, Lithuania, June 26-29.

[11] Riaz, Z., Edwards, D.J., Thorpe, A. (2006) SightSafety: a hybrid information and communication technology system for reducing vehicle/pedestrian collisions, Automation in Construction, Vol. 15, No. 6, 719-728. 
[12] Teizer, J., Mantripragada, U., Venugopal, M. (2008) Analyzing the travel patterns of construction workers, Proceedings of the 25th International Symposium on Automation and Robotics in Construction - ISARC 2008, June 26-29, Vilnius, Lithuane.

[13] Wang, A. P., Chen, J. C., Hsu, P. L. (2004) Intelligent CAN-based Automotive Collision Avoidance warning system, Proceedings of the 2004 IEEE International Conference on Networking, Sensing \& Control, March 21-23, Taipei, Taiwan.

[14] Howden, N., Curmi, J., Heinze, C., Goss, S., and Murphy, G. (2003) Operational Knowledge Representation: Behaviour Capture, Modelling and Verification, Proceedings of the Eighth International Conference on Simulation Technology and Training (SimTecT '03), Adelaide Australia. 